\title{
Phase-field model for Hele-Shaw flows with arbitrary viscosity contrast. I. Theoretical approach
}

\author{
R. Folch, J. Casademunt, and A. Hernández-Machado \\ Departament d'Estructura i Constituents de la Matèria, Universitat de Barcelona, Avinguda Diagonal, 647, E-08028 Barcelona, Spain \\ L. Ramírez-Piscina \\ Departament de Física Aplicada, Universitat Politècnica de Catalunya, Avinguda Dr. Marañón, 50, E-08028 Barcelona, Spain
}

(Received 24 February 1999)

\begin{abstract}
We present a phase-field model for the dynamics of the interface between two inmiscible fluids with arbitrary viscosity contrast in a rectangular Hele-Shaw cell. With asymptotic matching techniques we check the model to yield the right Hele-Shaw equations in the sharp-interface limit, and compute the corrections to these equations to first order in the interface thickness. We also compute the effect of such corrections on the linear dispersion relation of the planar interface. We discuss in detail the conditions on the interface thickness to control the accuracy and convergence of the phase-field model to the limiting Hele-Shaw dynamics. In particular, the convergence appears to be slower for high viscosity contrasts. [S1063-651X(99)08208-2]

PACS number(s): 47.54.+r, 47.20.Hw, 05.90.+m, 05.10.-a
\end{abstract}

\section{INTRODUCTION}

The dynamics of morphologically unstable interfaces is a major problem in nonequilibrium physics from both fundamental and applied points of view. Relevant examples of those are dendritic growth, directional solidification, flows in porous media, flame propagation, electrodeposition, or bacterial growth [1]. The so-called Saffman-Taylor problem has played a central role in this context because of its relative simplicity both experimentally and in its theoretical formulation $[1,2]$. It deals with the motion of the interface between two inmiscible fluids within a Hele-Shaw cell. Due to the highly nonlinear and nonlocal nature of the interfacial dynamics of such systems, analytical understanding is scarce and restricted to high viscosity contrast [3], so in general one relies mostly on numerical work [4-9].

From a mathematical point of view, such systems are referred to as moving boundary problems. In practice this implies that one has to keep track of the interface where boundary conditions are applied, and solve a (linear) problem in the bulk which determines in turn the motion of the boundary. This kind of problem has traditionally been addressed in terms of boundary integral methods which reduce the dynamics of the interface to integrodifferential equations. The numerical integration of these equations is quite involved, however, particularly for long times, due to stiffness and numerical instability of the equations. In the case of HeleShaw flows, boundary integral methods have successfully been applied [6-9], although quite sophisticated algorithms have usually been necessary [9].

Recently, so-called phase-field equations have been proposed in the context of solidification problems as a different approach to the interface dynamics [10-19]. In the spirit of the well known time-dependent Ginzburg-Landau models [20], the method avoids the tracking of the interface by introducing an auxiliary field (analogous to an order parameter) which locates the interface and whose dynamics is coupled to the other physical fields through an appropriate set of partial differential equations. In this way, there is no boundary condition to apply explicitly at the interface and the whole system is treated as bulk.

This method introduces a mesoscale $\epsilon$, which is not present in the original macroscopic equations and gives a finite thickness to the interface. The equations are then chosen in such a way that the original bulk equations and boundary conditions are recovered in the $\epsilon \rightarrow 0$ limit. Therefore, the phase-field equations for a given model are not intended to describe the true mesoscale physics of the system, and are then not unique. In fact there is considerable freedom in choosing a particular form of them, with criteria of either numerical efficiency and convergence [13] or other physical criteria such as thermodynamic consistency [14]. In any case, the nature of the phase-field approach is completely different from the sharp-interface models, and therefore the actual numerical advantages and limitations of both are also quite distinct. This makes the two approaches complementary and competitive in different physical situations. A remarkable advantage of the phase-field approach is that it is much simpler to implement satisfactorily from a numerical point of view. On the other hand, the phase-field approach is usually more amenable to generalization, in the sense that it allows one to introduce variations and new elements without any major modification of the numerical scheme, for instance in the treatment of fluctuations, liquid crystals [18], and other complex fluids [9]. Finally, the phase-field approach can handle very naturally situations where the sharp interface model is not appropriate, such as for instance topology changes like interface pinching leading to the breakup of bubbles.

In this paper we introduce a phase-field model for HeleShaw flows with arbitrary viscosity contrast (or Atwood ratio) $c=\left(\mu_{1}-\mu_{2}\right) /\left(\mu_{1}+\mu_{2}\right)$. Although in the high contrast limit $c=1$ the Hele-Shaw dynamics is quite analogous to the one-sided solidification problem (in the appropriate approximations [8]), the arbitrary viscosity contrast case has been shown to exhibit quite different dynamics than solidification problems, and has in fact opened some interesting questions, particularly concerning the sensitivity of finger competition 
to viscosity contrast $[4-7,21]$ and the long time asymptotics of the low viscosity contrast limit [7].

The model presented here is inspired in the vortex-sheet formulation of the problem [4], in which the relevant dynamic variable in the bulk is the stream function. Similar ideas have previously been applied to describe physically diffuse interfaces in the context of steady state selection in thermal plumes [22]. Usually phase-field models are naturally suited to symmetric situations (two-sided models). The present case of Hele-Shaw flow is no exception and becomes most efficient for $c=0$. Finite $c$ can also be handled but the model becomes computationally inefficient in the limit $c$ $\rightarrow 1$, since this limit must be taken formally after the $\epsilon \rightarrow 0$ limit. A phase-field model for this one-sided case must differ essentially from the one presented here, such as in the spirit of Ref. [19].

The layout of the rest of the paper is as follows: in Sec. II A we recall the Hele-Shaw macroscopic equations in terms of the stream function, whereas in Sec. II B we present our phase-field equations. We then show in Sec. III that the phase-field equations reduce to the macroscopic ones in the sharp-interface limit. Deviations from that limiting behavior are derived from the phase-field equations themselves to first order in the interface thickness in Sec. IV, and their effect on the linear regime is computed in Sec. V. Finally, a brief summary is given in Sec. VI.

\section{MODEL}

We consider the general case of an interface with surface tension $\sigma$ between two fluids with distinct viscosities $\left(\mu_{1}, \mu_{2}\right)$ and densities $\left(\rho_{1}, \rho_{2}\right)$ moving in a rectangular Hele-Shaw cell of width $W$ ( $x$ direction) and gap $b$ ( $z$ direction), under an effective gravity $g_{\text {eff }}$ (negative $y$ direction) and with an injection velocity $V_{\infty}$ (positive $y$ direction). Label 1 (2) corresponds to the upper (lower) fluid.

\section{A. Macroscopic equations}

Darcy's law is assumed to hold for each fluid, thus defining a certain velocity potential in each bulk, but not on the interface. In contrast, the bulk incompressibility and the continuity of normal velocities on the interface allow us to define its harmonic conjugate, the stream function $\psi$, even on the interface through $u_{x}=\partial_{y} \psi, u_{y}=-\partial_{x} \psi$, where $u_{x}$ and $u_{y}$ are the $x$ and $y$ components of the fluid velocity field $\vec{u}$. Then Darcy's law results in a Laplace equation for the stream function (potential flow) and a certain jump for the tangential fluid velocities on the interface, whose value takes into account the Gibbs-Thomson relationship. The fact that the stream function is continuous at the interface makes the use of this variable particularly convenient. The Hele-Shaw equations in stream function formulation [4] can be written in dimensionless form as

$$
\begin{gathered}
\nabla^{2} \psi=0 \\
\psi_{r}\left(0^{+}\right)-\psi_{r}\left(0^{-}\right)=-\gamma-c\left[\psi_{r}\left(0^{+}\right)+\psi_{r}\left(0^{-}\right)\right], \\
\psi_{s}\left(0^{+}\right)=\psi_{s}\left(0^{-}\right)=-\mathrm{v}_{n} .
\end{gathered}
$$

Here $r$ is a coordinate normal to the interface and with origin on it, positive in fluid $1\left(0^{ \pm}\right.$then means on the interface coming from each side), $s$ is the arclength along the interface, and the unit vectors satisfy $\hat{s} \times \hat{r}=\hat{x} \times \hat{y}$, the subscripts stand for partial derivatives except for $\mathrm{v}_{n}(s)$, which is the normal velocity of the interface, positive towards fluid 1 , and

$$
\frac{\gamma(s)}{2} \equiv B \kappa_{s}+\hat{y} \cdot \hat{s}
$$

with $\kappa(s)$ the interface curvature, positive for a bump into fluid 2. The dynamics are controlled by the two dimensionless parameters

$$
B=\frac{b^{2} \sigma}{12 W^{2}\left[V_{\infty}\left(\mu_{1}-\mu_{2}\right)+g_{\text {eff }} \frac{b^{2}}{12}\left(\rho_{1}-\rho_{2}\right)\right]}, \quad c=\frac{\mu_{1}-\mu_{2}}{\mu_{1}+\mu_{2}} .
$$

We will not be interested in negative values of $B$ (stable configuration) nor $c$ (mirror image interface of $-c$ ). Thus $B$ is a dimensionless surface tension, and can be understood as the ratio between the capillary (stabilizing) force and the driving (destabilizing) force (injection plus gravity), and $c$ is the viscosity contrast, which is so far completely arbitrary: $0 \leqslant c \leqslant 1$. This corresponds to having set ourselves in the frame moving with the fluid at infinity (or, equivalently, with the mean interface) and taken $W$ as the unit length and $U_{*}$ $\equiv c V_{\infty}+g_{\text {eff }}\left[b^{2}\left(\rho_{1}-\rho_{2}\right) / 12\left(\mu_{1}+\mu_{2}\right)\right]$ as the unit velocity (see Ref. [4]).

Note that Eqs. (2.1) and (2.2) can be written together as

$$
\nabla^{2} \psi=-w, \quad w=\left\{\gamma(s)+c\left[\psi_{r}\left(0^{+}\right)+\psi_{r}\left(0^{-}\right)\right]\right\} \delta(r),
$$

where $\delta(r)$ is the Dirac $\delta$ distribution and $w \equiv \hat{z} \cdot(\vec{\nabla} \times \vec{u})$ is the fluid vorticity, which is confined to the interface.

\section{B. Phase-field equations}

We put forward the following phase-field model for the above equations with $\theta$ being the phase field:

$$
\begin{gathered}
\epsilon \frac{\partial \psi}{\partial t}=\nabla^{2} \psi+c \vec{\nabla} \cdot(\theta \vec{\nabla} \psi)+\frac{1}{\epsilon} \frac{1}{2 \sqrt{2}} \gamma(\theta)\left(1-\theta^{2}\right), \\
\epsilon^{2} \frac{\partial \theta}{\partial t}=f(\theta)+\epsilon^{2} \nabla^{2} \theta+\epsilon^{2} \kappa(\theta)|\vec{\nabla} \theta|+\epsilon^{2} \hat{z} \cdot(\vec{\nabla} \psi \times \vec{\nabla} \theta),
\end{gathered}
$$

where $f(\theta) \equiv \theta\left(1-\theta^{2}\right)$, and $\gamma(\theta) / 2 \equiv \hat{s}(\theta) \cdot(B \vec{\nabla} \kappa(\theta)+\hat{y})$, $\kappa(\theta) \equiv-\vec{\nabla} \cdot \hat{r}(\theta)$, with $\hat{r}(\theta) \equiv \vec{\nabla} \theta /|\vec{\nabla} \theta|$ and $\hat{s}(\theta) \equiv \hat{r}(\theta)$ $\times \hat{z}$, together with the boundary condition

$$
\theta(y \rightarrow \pm \infty)= \pm 1
$$

so that $\theta=+1(-1)$ corresponds to fluid $1(2) . \gamma(\theta)$ and $\kappa(\theta)$ are functionals which generalize the magnitudes defined above for the interface, now to any level set of the phasefield. 
If we leave the two last terms aside, Eq. (2.8) is the CahnHilliard equation for a nonconserved order parameter or model $A$ (without noise) in the classification of Ref. [20] of time-dependent Ginzburg-Landau models. The field in this model is known to relax towards a kink solution of a certain width in a short time scale, and then to evolve to minimize the length of the effective interface according to Allen-Cahn law (i.e., with normal velocity proportional to the local curvature). The factor multiplying the laplacian has been choosen to be $\epsilon^{2}$ for the kink width to be $O(\epsilon)$, so that $\epsilon$ can be considered the interface thickness, i.e., the small parameter in the asymptotic analysis that will be performed in Sec. III. On the other hand, the $\epsilon^{2}$ factor in the time derivative ensures that relaxation toward the kink is much faster than the evolution of the interface. Notice that model $A$ describes the relaxational dynamics of a nonconserved order parameter, whereas our problem is actually nonrelaxational and strictly conserved (mass conservation and inmiscibility). The other two term in the phase-field equation will correct this apparent contradiction. In order to cancel out the local AllenCahn dynamics of the interface which is built in model $A$, we add the term $\epsilon^{2} \kappa(\theta)|\vec{\nabla} \theta|$. It will be shown that such term cancels out Allen-Cahn law by giving rise, to leading order, to an identical contribution but with opposite sign. With these elements so far, our phase field relaxes to a kink profile located along an arbitrary interface which (if sufficiently smooth) remains almost completely stationary, regardless of its shape. This is because the dynamical effect of surface tension associated with the Ginzburg-Landau free energy has been removed (up to first order), and the interface has not yet been coupled to the fluid flow, represented by the stream function. This coupling is achieved by adding the last term in Eq. (2.8), which stands for $-\epsilon^{2} \vec{u} \cdot \vec{\nabla} \theta$, and thus sets the phase field-and therefore the interface-in the frame moving with the fluid velocity $\vec{u}$. This term restores the fully nonlocal dynamics of the Hele-Shaw model. In particular it yields the continuity of normal velocities [Eq. (2.3)], and reintroduces surface tension, which is contained in the dynamical equation for the stream function through $\gamma(\theta)$.

As for Eq. (2.7), its right hand side is intended to reproduce Eq. (2.6), and therefore also Eqs. (2.1) and (2.2). If the phase field $\theta$ has a kink shape, $1-\theta^{2}$ is a peaked function which, when divided by $\epsilon$, gives rise to the $\delta$ distribution for the vorticity. However, this only accounts for the $\gamma$ in the weight of the $\delta$. The part proportional to the viscosity contrast $c$ must be put apart as the $c \vec{\nabla} \cdot(\theta \vec{\nabla} \psi)$ term because of the nonlocal character of $\psi_{r}\left(0^{+}\right)+\psi_{r}\left(0^{-}\right)$. Finally, the time derivative is multiplied by $\epsilon$ to recover the Laplacian (and not diffusive) behavior of the Hele-Shaw flow in the sharpinterface limit.

In spite of important differences, the proposed phase field equations (2.7) and (2.8) contain certain similarities to the problem of a thermal plume in a Hele-Shaw cell under gravity [22]. In such a problem there is only one fluid heated from the center of the channel. The heat diffuses toward the lateral walls, but the temperature profile is not linear, since the fluid density and viscosity decrease with temperature, so that the fluid in the middle of the channel raises because of buoyancy. As a result a so-called plume of hot fluid with a shape similar to the Saffman-Taylor finger, with a stationary upward velocity, and a width close to $\frac{1}{2}$ is formed. Outside the plume the fluid is colder, and the transition between the two zones is relatively abrupt, so that one can think in terms of an interface of a certain small thickness. Thus the equation for the phase field [Eq. (2.8)] could be thought of as a diffusion equation for the temperature in a thermal plume. However, the available equations for that problem hold only for the steady state [22], whereas our phase-field model is intended to describe the whole dynamics. Generalization of the thermal plume equations to include the dynamics is not trivial for nonvanishing viscosity contrast. As a matter of fact, Ref. [22] must restrict itself to low viscosity contrasts - as is the case in thermal plumes-whereas we formulate the model for arbitrary viscosity contrast. An interesting difference is the term $\epsilon^{2} \kappa(\theta)|\vec{\nabla} \theta|$ cancelling out Allen-Cahn law. The absence of that term in the thermal plume equations does not prevent the Hele-Shaw steady state equations from being recovered in the sharp-interface limit because of the lower power of $\epsilon$ used in the $\vec{u} \cdot \vec{\nabla} \theta$ term, but then Allen-Cahn law arises in the corrections at first order in the interface thickness. In contrast, by means of this $\epsilon^{2} \kappa(\theta)|\vec{\nabla} \theta|$ term we achieve cancellation of the Allen-Cahn law even in such corrections, as we will see in Sec. IV. Finally, another major difference in the case of thermal plumes is the absence of surface tension.

\section{SHARP-INTERFACE LIMIT}

In order to analyze the small- $\epsilon$ behavior of the phase-field equations (2.7) and (2.8), we expand their fields in powers of $\epsilon$. The expected abrupt variations of these fields through the interface will make it necessary to perform two different expansions. In the interface region (inner region) we rescale the differential operators appearing in these phase-field equations by rewriting them in terms of the stretched normal coordinate $\rho \equiv r / \epsilon$ (see the Appendix). The expansions in the inner region will be matched order by order in powers of $\epsilon$ to those in the outer region (in the bulk far from the interface), where the coordinates are not rescaled. The outer and inner expansions are written, respectively, as

$$
a(r, s, t)=a_{0}(r, s, t)+\epsilon a_{1}(r, s, t)+\epsilon^{2} a_{2}(r, s, t)+\cdots,
$$

$$
A(\rho, s, t)=A_{0}(\rho, s, t)+\epsilon A_{1}(\rho, s, t)+\epsilon^{2} A_{2}(\rho, s, t)+\cdots,
$$

where capital letters denote fields written in terms of the rescaled coordinate. This results in the following matching conditions:

$$
\begin{gathered}
A_{0}(\rho, s, t)=a_{0}\left(0^{ \pm}, s, t\right), \\
A_{1}(\rho, s, t)=a_{1}\left(0^{ \pm}, s, t\right)+\rho a_{0, r}\left(0^{ \pm}, s, t\right) \text { as } \rho \rightarrow \pm \infty \\
A_{2}(\rho, s, t)=a_{2}\left(0^{ \pm}, s, t\right)+\rho a_{1, r}\left(0^{ \pm}, s, t\right)+\frac{\rho^{2}}{2} a_{0, r r}\left(0^{ \pm}, s, t\right), \\
\cdots .
\end{gathered}
$$


Therefore,

$$
\begin{gathered}
A_{0, \rho}( \pm \infty, s, t)=A_{1, \rho \rho}( \pm \infty, s, t)=\cdots=0 \\
A_{1, \rho}( \pm \infty, s, t)=a_{0, r}\left(0^{ \pm}, s, t\right) \\
A_{2, \rho}(\rho, s, t)=a_{1, r}\left(0^{ \pm}, s, t\right)+\rho a_{0, r r}\left(0^{ \pm}, s, t\right) \quad \text { as } \rho \rightarrow \pm \infty \\
\cdots
\end{gathered}
$$

In practice, one does not find explicit solutions for the fields, but some set of equations for them. A sharp-interface model for the small- $\epsilon$ dynamics of the phase-field equations (2.7) and (2.8) is then given by the set of equations obeyed by the outer fields: Those obtained at lowest order in the interface thickness $\epsilon\left(O\left(\epsilon^{0}\right)\right)$ constitute the $\epsilon \rightarrow 0$ limit of the phase-field model, which we carry out in this section; whereas those obtained up to $O(\epsilon)$ represent what we will call (following Karma and Rappel [13]) a "thin-interface" model, a model keeping finite interface thickness effects, such as the one derived in Sec. IV.

\section{A. Outer equations}

Straightforward substitution of the outer expansion [Eq. (3.1)] in the outer equations, (2.7) and (2.8), will yield the bulk fields: a functional dependence for the phase-field and a differential equation for the stream function.

Equation (2.8) reads

$$
\begin{gathered}
f_{0}(\theta)=f\left(\theta_{0}\right)=0 \Rightarrow \theta_{0}=0, \pm 1=\text { const } \quad \text { at } O\left(\epsilon_{0}\right), \\
f_{1}(\theta)=-2 \theta_{1}=0 \Rightarrow \theta_{1}=0 \quad \text { at } O(\epsilon),
\end{gathered}
$$

and iterating, we obtain

$$
\theta_{i}=0 \quad \forall i>0 .
$$

Due to Eqs. (3.5) and (3.7), $\theta= \pm 1$ to all orders, and, therefore, the $\left(1-\theta^{2}\right)$ term in Eq. (2.7) does not enter this outer limit, whereas the viscosity contrast term in that equation becomes $\pm c \nabla^{2} \psi$, depending on the phase. Hence Eq. (2.7) in this outer region reads

$$
\epsilon \frac{\partial \psi}{\partial t}=(1 \pm c) \nabla^{2} \psi
$$

which implies

$$
\nabla^{2} \psi_{0}=0, \quad \frac{\partial \psi_{i}}{\partial t}=(1 \pm c) \nabla^{2} \psi_{i+1}, \quad \forall i \geqslant 0,
$$

except for $c=1$. Note that we have recovered the sharpinterface equation (2.1) in the $\epsilon \rightarrow 0$ limit. For $c=1$, Eq. (2.1) is still recovered in the +1 phase (viscous fluid), whereas in the -1 phase (inviscid fluid) the stream function turns out to be constant in time to all orders. Although the inviscid fluid does not enter the problem in this limit [see Eq. (2.2)], it still has a nontrivial dynamics, since the stream function in it must evolve to keep satisfying Eq. (2.3), and therefore, strictly speaking, we do not really get the right sharpinterface limit for $c$ exactly equal to 1 . However, the model can be applied to physical high viscosity contrast pairs of fluids. We shall come back to this point in Sec. IV.

\section{B. Inner equations}

In turn, the interface boundary conditions for the stream function are given by the leading-order outer quantities $\psi_{0, s}\left(0^{ \pm}\right)$and $\psi_{0, r}\left(0^{+}\right)-\psi_{0, r}\left(0^{-}\right)$. According to the matching conditions (3.3) and (3.4), these equal the inner ones $\Psi_{0, s}( \pm \infty)$ and $\Psi_{1, \rho}(+\infty)-\Psi_{1, \rho}(-\infty)$, respectively. Because of the specific structure of our phase-field equations, (2.7) and (2.8), we will need the first two orders in the inner version of Eq. (2.8) and the lowest one in that of Eq. (2.7) to obtain $\Psi_{0, s}( \pm \infty)$, and the two first in Eq. (2.7) and the lowest in Eq. (2.8), to obtain $\Psi_{1, \rho}(+\infty)-\Psi_{1, \rho}(-\infty)$. Therefore, we compute the two first orders in both Eqs. (2.8) and (2.7), by substituting the inner expansion (3.2) in the inner (rescaled) equations (all whose terms are derived in the Appendix):

Equation (2.8) up to $O(\epsilon)$ reads [see Eqs. (3.2), (A10), (A11), and (A19)]

$$
\begin{aligned}
-\epsilon \mathrm{\vee}_{n} \Theta_{0, \rho}= & f\left(\Theta_{0}\right)+\epsilon \Theta_{1} f^{\prime}\left(\Theta_{0}\right)+\Theta_{0, \rho \rho}+\epsilon \Theta_{1, \rho \rho} \\
& +\epsilon\left(\Theta_{0, \rho} \Psi_{0, s}-\Theta_{0, s} \Psi_{0, \rho}\right) .
\end{aligned}
$$

Its $O\left(\epsilon^{0}\right)$ part,

$$
f\left(\Theta_{0}\right)+\Theta_{0, \rho \rho}=0,
$$

together with the boundary conditions specified by the matching [Eqs. (3.3) and (3.4)] with the outer expansion Eq. (3.5), gives the so-called kink solution

$$
\Theta_{0}=\tanh \frac{\rho}{\sqrt{2}} \Rightarrow \Theta_{0, \rho}=\frac{1}{\sqrt{2}} \operatorname{sech}^{2} \frac{\rho}{\sqrt{2}}=\frac{1}{\sqrt{2}}\left(1-\Theta_{0}^{2}\right) .
$$

Hence we find the $\Theta_{0, s}$ term to vanish, and Eq. (3.10) reads, at $O(\epsilon)$,

$$
-\mathrm{v}_{n} \Theta_{0, \rho}=\Theta_{1} f^{\prime}\left(\Theta_{0}\right)+\Theta_{1, \rho \rho}+\Theta_{0, \rho} \Psi_{0, s} .
$$

As for Eq. (2.7), it reads, up to $O(1 / \epsilon)$ [see Eqs. (3.2), (A9), (A10), (A12), and (A17)],

$$
\begin{aligned}
\frac{1}{\epsilon^{2}} \Psi_{0, \rho \rho} & +\frac{1}{\epsilon}\left(\Psi_{1, \rho \rho}-\kappa \Psi_{0, \rho}\right)+c\left\{\frac{1}{\epsilon^{2}}\left(\Theta_{0} \Psi_{0, \rho}\right)_{\rho}\right. \\
+ & \left.\frac{1}{\epsilon}\left[\left(\Theta_{0} \Psi_{1, \rho}\right)_{\rho}+\left(\Theta_{1} \Psi_{0, \rho}\right)_{\rho}-\kappa \Theta_{0} \Psi_{0, \rho}\right]\right\} \\
+ & \frac{1}{\epsilon} \frac{1}{2 \sqrt{2}} \gamma\left(1-\Theta_{0}^{2}\right)=0 .
\end{aligned}
$$

From its $O\left(1 / \epsilon^{2}\right)$ part, we know that

$$
\Psi_{0, \rho}\left(1+c \Theta_{0}\right)=\text { const. }
$$


Since $\Psi_{0, \rho}$ has no correspondence with the outer expansion, it must vanish at infinity [Eq. (3.4)]. Then we know the constant to be zero. Now, since the term in brackets vanishes only for $c=1$ and $\rho \rightarrow-\infty$, we deduce that

$$
\Psi_{0, \rho}=0 .
$$

We then put Eq. (3.16) into Eq. (3.14) at $O(1 / \epsilon)$ :

$$
\Psi_{1, \rho \rho}+c\left(\Theta_{0} \Psi_{1, \rho}\right)_{\rho}=-\frac{\gamma}{2} \Theta_{0, \rho} .
$$

Finally, Eqs. (3.13) and (3.17) will yield the macroscopic equations (2.3) and (2.2), respectively: Eq. (3.13) can be rewritten in the form

$$
\hat{L} \Theta_{1} \equiv\left[f^{\prime}\left(\Theta_{0}\right)+\frac{\partial^{2}}{\partial \rho^{2}}\right] \Theta_{1}=-\Theta_{0, \rho}\left(\mathrm{v}_{n}+\Psi_{0, s}\right) .
$$

We realize that $\hat{L} \Theta_{0, \rho}$ equals the partial derivative with respect to $\rho$ of Eq. (3.11), which, in turn, vanishes. Hence we write the solvability condition

$$
\int_{-\infty}^{+\infty}\left(\mathrm{v}_{n}+\Psi_{0, s}\right) \Theta_{0, \rho}^{2} d \rho=0 .
$$

Using Eq. (3.16) we know that $\Psi_{0, \rho s}=0=\Psi_{0, s \rho}$, and can take $\Psi_{0, s}$ out of the integral as well as $\mathrm{v}_{n}$. Since the quantity left under the integral sign $\left(\Theta_{0, \rho}\right)^{2}$ is always positive, we find that $\mathrm{v}_{n}+\Psi_{0, s}$ must vanish, and, matching with the outer expansion, we obtain Eq. (2.3) for $\psi_{0}$.

On the other hand, integrating Eq. (3.17) with respect to $\rho$, we obtain

$$
\Psi_{1, \rho}=-\frac{\gamma}{2} \Theta_{0}-c \Theta_{0} \Psi_{1, \rho}+a_{1}(s),
$$

where $a_{1}(s)$ is an arbitrary function of $s$. Computing $\Psi_{1, \rho}(+\infty)-\Psi_{1, \rho}(-\infty)$ and matching with the outer expansion [Eq. (3.4)] gives Eq. (2.2) for $\psi_{0}$. This completes the sharp-interface limit.

\section{FIRST ORDER CORRECTIONS TO THE SHARP-INTERFACE LIMIT}

In the phase-field model the interface width and the convergence to the sharp-interface limit is controlled by the small but finite value of the parameter $\epsilon$. Then, the question of which value of $\epsilon$ is needed to reproduce the actual HeleShaw dynamics accurately for given values of the physical parameters $B$ and $c$ arises. This question can be qualitatively answered by noting the distinct roles played by $\epsilon$ in the phase-field equations (2.7) and (2.8).

The $\epsilon$ factors appearing in $\epsilon^{2} \nabla^{2} \theta, \epsilon^{2} \kappa(\theta)|\vec{\nabla} \theta|$ and $(1 / \epsilon)(1 / 2 \sqrt{2}) \gamma(\theta)\left(1-\theta^{2}\right)$ all stand for the interface thickness, and this is required to be small compared to the longitudinal length scale $|k|^{-1}$ of the interface: $\epsilon|k| \ll 1$. In contrast, the $\epsilon$ in $\epsilon(\partial \psi / \partial t)$ has nothing to do with the interface thickness (and we will therefore denote it by $\widetilde{\epsilon}$ from now on), but its aim is to ensure that the stream function is Laplacian and not diffusive in the $\widetilde{\epsilon} \rightarrow 0$ limit, which commutes with the $\epsilon \rightarrow 0$ one [the reader can convince himself of this by going through the limit again but now considering $\widetilde{\epsilon}$ of $\left.O\left(\epsilon^{0}\right)\right]: \widetilde{\boldsymbol{\epsilon}}$ sets the time scale of the diffusion of the stream function through a given characteristic length of wave number $k, \tilde{\epsilon} /(1 \pm c) k^{2}$ [see Eq. (3.8)], which must be much smaller than the characteristic growth rate of the interface $|\omega|^{-1}$, so that the stream function is slaved to the interface: $\widetilde{\boldsymbol{\epsilon}}|\omega| / k^{2} \ll 1 \pm c$. We also realize that the viscosity contrast $c$ can be arbitrarily raised, as long as $\widetilde{\epsilon}$ is correspondingly lowered. So our model is valid even for $c \rightarrow 1$, as long as this limit is taken formally after the $\epsilon \rightarrow 0$ one.

The $\epsilon^{2}$ in $\epsilon^{2}(\partial \theta / \partial t)$ represents the relaxation time of the phase field toward the steady kink solution [see Eq. (2.8)], which must be kept well below the interface growth time $|\omega|^{-1}$ for the phase field to remain close to the kink profile during the interface evolution: $\epsilon^{2}|\omega| \ll 1$. This factor must be the same that the one in $\epsilon^{2} \hat{z} \cdot(\vec{\nabla} \psi \times \vec{\nabla} \theta)$ in order to obtain the macroscopic equation (2.3). In fact there are at least two distinct powers of $\epsilon$ for this relaxation time $\left(\epsilon\right.$ and $\left.\epsilon^{2}\right)$ for which the right sharp-interface limit is achieved, and the corrections which we will compute would also be the same.

To sum up, there are at least two independent small parameters $(\epsilon$ and $\widetilde{\epsilon})$ controlling the limit. When trying to approach macroscopic solutions by means of numerical integration of the phase-field equations, it is very convenient to vary them independently in order to save computing time, since both affect it [23].

A more quantitative answer to the question of the necessary values of $\epsilon$ and $\widetilde{\epsilon}$ to obtain a given precision can be given by extending the asymptotic analysis of Sec. III to first order in the interface thickness $\epsilon$ considering $\widetilde{\epsilon}$ of $O(\epsilon)$. Thus we will obtain a thin-interface model containing the corrections to the limit up to that order in $\epsilon$ and $\widetilde{\epsilon}$.

According to the matching conditions (3.3), the corrections to the interface boundary conditions for the stream function at first order in $\epsilon, \psi_{1, s}\left(0^{ \pm}\right)$and $\psi_{1, r}\left(0^{+}\right)$ $-\psi_{1, r}\left(0^{-}\right)$, are to be identified as terms in the expansion of $\Psi_{1, s}( \pm \infty)$ and $\Psi_{2, \rho}(+\infty)-\Psi_{2, \rho}(-\infty)$, respectively. Now we will need the second order in Eq. (2.8) and the first in Eq. (2.7) to compute $\Psi_{1, s}( \pm \infty)$, and the second in Eq. (2.7) and the first in Eq. (2.8) to obtain $\Psi_{2, \rho}(+\infty)-\Psi_{2, \rho}(-\infty)$. Therefore, we must compute the next order both in Eqs. (2.8) and (2.7), but, first, we can still extract some information from the lower orders.

On the one hand, we found that $\Psi_{0, s}=-\mathrm{v}_{n}$. We put this into Eq. (3.13) to obtain the differential equation for $\Theta_{1}$,

$$
\Theta_{1} f^{\prime}\left(\Theta_{0}\right)+\Theta_{1, \rho \rho}=0
$$

with boundary conditions coming from the matching Eq. (3.3) with Eq. (3.7) $\Theta_{1}( \pm \infty)=\Theta_{1, \rho}( \pm \infty)=0$ and solution $\Theta_{1}=0$.

The integral with respect to $\rho$ of Eq. (3.20) is

$$
\Psi_{1}=-\frac{\gamma}{2} \int \frac{\Theta_{0} d \rho}{1+c \Theta_{0}}+a_{1}(s) \int \frac{d \rho}{1+c \Theta_{0}} .
$$


According to the matching equation (3.3), the $\rho \rightarrow \pm \infty$ asymptotics of $\Psi_{1}(\rho)$ should consist of a finite term $\psi_{1}\left(0^{ \pm}\right)$ and a diverging one $\rho \psi_{0, r}\left(0^{ \pm}\right)$. For vanishing viscosity contrast, the last integral in Eq. (4.2) is $\rho a_{1}(s)$, and clearly does not contribute to the finite term $\psi_{1}\left(0^{ \pm}\right)$. Then, since $\Theta_{0}$ is an odd function of $\rho$, its integral with respect to $\rho$ will be even, and $\psi_{1}\left(0^{+}\right)=\psi_{1}\left(0^{-}\right)$, i.e., the fluid velocity normal to the interface will be continous on it.

For nonzero values of $c$, however, one must compute the integrals in Eq. (4.2), find their $\rho \rightarrow \pm \infty$ asymptotics, and identify $\psi_{1}\left(0^{ \pm}\right)$and $\psi_{0, r}\left(0^{ \pm}\right)$. Requiring this latter quantity to be consistent with Eq. (2.2) for $\psi_{0}$, one fixes $a_{1}(s)$; putting this back into the identified $\psi_{1}\left(0^{ \pm}\right)$value, one finds

$$
\begin{aligned}
\psi_{1}\left(0^{ \pm}\right)= & -\frac{\sqrt{2}}{2}\left\{\gamma+c\left[\psi_{0, r}\left(0^{+}\right)+\psi_{0, r}\left(0^{-}\right)\right]\right\} \ln \frac{1 \pm c}{2} \\
& +a_{2}(s),
\end{aligned}
$$

where $a_{2}(s)$ is another arbitrary function of $s$. This will give rise to a discontinuity in the fluid velocity:

$$
\begin{aligned}
\psi_{1, s}\left(0^{+}\right)-\psi_{1, s}\left(0^{-}\right)= & -\frac{\sqrt{2}}{2}\left\{\gamma_{s}+c\left[\psi_{0, r s}\left(0^{+}\right)\right.\right. \\
& \left.\left.+\psi_{0, r s}\left(0^{-}\right)\right]\right\} \ln \frac{1+c}{1-c} \\
= & -c \sqrt{2}\left\{\gamma_{s}+c\left[\psi_{0, r s}\left(0^{+}\right)\right.\right. \\
& \left.\left.+\psi_{0, r s}\left(0^{-}\right)\right]\right\}+O\left(c^{3}\right) .
\end{aligned}
$$

In order to fix $\partial_{s} a_{2}(s)$, we compute the next order $\left(O\left(\epsilon^{2}\right)\right)$ of Eq. (2.8) to obtain [see Eqs. (3.2), (A10), (A11), and (A19)]

$$
\Theta_{2} f^{\prime}\left(\Theta_{0}\right)+\Theta_{2, \rho \rho}-\rho \kappa \mathrm{v}_{n} \Theta_{0, \rho}+\Psi_{1, s} \Theta_{0, \rho}=0 .
$$

This has the same structure as Eq. (3.13), and an analog solvability condition applies:

$$
\int_{-\infty}^{+\infty} \Psi_{1, s} \Theta_{0, \rho}^{2} d \rho=0
$$

Substitution of the expression for $\Psi_{1}$ obtained by performing the integrals in Eq. (4.2) into this condition and subsequent computation of the resulting integral fixes $\partial_{s} a_{2}(s)$ so that

$$
\begin{aligned}
\psi_{1, s}\left(0^{ \pm}\right)= & -\frac{\sqrt{2}}{2}\left[\frac{\gamma_{s}}{2}+c \frac{\psi_{0, r s}\left(0^{+}\right)+\psi_{0, r s}\left(0^{-}\right)}{2}\right] \\
& \times\left[1-\frac{1}{c^{2}}+\left( \pm 1+\frac{\left(1 / c^{3}\right)-(3 / c)}{2}\right) \ln \frac{1+c}{1-c}\right] \\
= & \sqrt{2}\left[\frac{\gamma_{s}}{2}+c \frac{\psi_{0, r s}\left(0^{+}\right)+\psi_{0, r s}\left(0^{-}\right)}{2}\right] \\
& \times\left[\frac{5}{6} \mp c+\frac{2}{5} c^{2}+O\left(c^{3}\right)\right] .
\end{aligned}
$$

Finally, to obtain $\Psi_{2, \rho}(+\infty)-\Psi_{2, \rho}(-\infty)$, we need Eq. (2.7) at $\left(O\left(\epsilon^{0}\right)\right)$ [see Eqs. (3.2), (A9), (A10), (A12), and (A17)]:

$$
\begin{aligned}
& \Psi_{2, \rho \rho}-\kappa \Psi_{1, \rho}-\partial_{s} \mathrm{v}_{n}+c\left[\left(\Theta_{0} \Psi_{2, \rho}\right)_{\rho}-\kappa \Psi_{1, \rho} \Theta_{0}-\partial_{s} \mathrm{v}_{n} \Theta_{0}\right] \\
& \quad+3 B \rho \kappa \kappa_{s} \Theta_{0, \rho}=0 .
\end{aligned}
$$

Integrating this from $\rho \rightarrow-\infty$ to $\rho \rightarrow+\infty$, we obtain

$$
\begin{aligned}
& {\left[\Psi_{2, \rho}\right]_{-\infty}^{+\infty}-\kappa \int_{-\infty}^{+\infty}\left(1+c \Theta_{0}\right) \Psi_{1, \rho} d \rho-\partial_{s} \mathrm{v}_{n}[\rho]_{-\infty}^{+\infty}} \\
& +c\left[\Theta_{0} \Psi_{2, \rho}\right]_{-\infty}^{+\infty}=0,
\end{aligned}
$$

where we have omitted integrals of odd functions of $\rho$. We use Eq. (3.20) to rewrite the integrand of the remaining integral as $-(\gamma / 2) \Theta_{0}+a_{1}(s) . \Theta_{0}$ is an odd function of $\rho$ and does not contribute to the integral, whereas $a_{1}(s)$ gives rise to a divergent term of the type $[\rho]_{-\infty}^{+\infty}$. According to the matching equation (3.4), $\psi_{1, r}\left(0^{ \pm}\right)$corresponds to the finite part of $\Psi_{2, \rho}( \pm \infty)$, so that we find

$$
\psi_{1, r}\left(0^{+}\right)-\psi_{1, r}\left(0^{-}\right)=-c\left[\psi_{1, r}\left(0^{+}\right)+\psi_{1, r}\left(0^{-}\right)\right],
$$

which will leave the jump of the normal derivative of the stream function across the interface unaffected at first order in the kink width.

Putting Eqs. (3.8), (2.3), and (2.2) for $\psi_{0}$, and Eqs. (4.7) and (4.10) together, we obtain an effective sharp-interface model for the dynamics of the $\theta=0$ level set up to first order in $\epsilon$ and $\widetilde{\epsilon}$ :

$$
\begin{gathered}
\tilde{\epsilon} \frac{\partial \psi}{\partial t}=(1 \pm c) \nabla^{2} \psi, \\
\psi_{r}\left(0^{+}\right)-\psi_{r}\left(0^{-}\right)=-\Gamma, \\
\psi_{s}\left(0^{ \pm}\right)=-\mathrm{v}_{n}-\epsilon \frac{\sqrt{2}}{2} \frac{\Gamma_{s}}{2} g_{ \pm}(c) \\
=-\mathrm{v}_{n}+\epsilon \sqrt{2} \frac{\Gamma_{s}}{2}\left[\frac{5}{6} \mp c+\frac{2}{5} c^{2}+O\left(c^{3}\right)\right],
\end{gathered}
$$

where $\Gamma \equiv \gamma+c\left[\psi_{r}\left(0^{+}\right)+\psi_{r}\left(0^{-}\right)\right]$is the weight of the vorticity defined in Eq. (2.6) evaluated up to $O(\epsilon)$ and

$$
g_{ \pm}(c)=1-\left(1 / c^{2}\right)+\left( \pm 1+\frac{\left(1 / c^{3}\right)-(3 / c)}{2}\right) \ln \frac{1+c}{1-c} .
$$

Note that the desired corrections to the limiting equations (2.1)-(2.3) in Eqs. (4.11) and (4.13) go as $\widetilde{\epsilon}$ and $\epsilon$, respectively, and the fact that Eq. (2.2) remains unaffected. Note as well that the correction in $\epsilon$ appearing in Eq. (4.13) has nothing to do with an Allen-Cahn law. So the $\epsilon^{2} \kappa(\theta)|\vec{\nabla} \theta|$ term has cancelled this out even in the first order corrections.

\section{LINEAR DISPERSION RELATION UP TO FIRST ORDER IN THE INTERFACE THICKNESS}

In order to see how such corrections affect some relevant specific situation, we compute the linear dispersion relation of a perturbation to the planar interface $y(x)=A e^{\omega t+i k x}$ for Eqs. (4.11)-(4.13). We make the ansatz 


$$
\psi(x, y)=a_{ \pm} A e^{\omega t+i k x-q_{ \pm}|y|},
$$

inspired by the actual Hele-Shaw result, where now the coefficient $a_{ \pm}$allows for distinct amplitudes in each phase for the stream function to satisfy the discontinuity in the normal velocities of Eq. (4.13), whereas the decay length $q_{ \pm}$in the $y$ direction is set not only by the wavelength of the perturbation $2 \pi / k$, but also by the diffusion length in Eq. (4.11), which is also different in each phase. Thus Eq. (4.11) yields

$$
q_{ \pm}=|k| p_{ \pm}, \quad p_{ \pm}=+\sqrt{1+\frac{\tilde{\epsilon} \omega}{k^{2}(1 \pm c)}} .
$$

In turn, taking into account that $\mathrm{v}_{n}=\omega A e^{\omega t+i k x}$ and $\gamma$ $=2 i A \operatorname{sgn}(k) w_{0} e^{\omega t+i k x}$ - where $\omega_{0}=|k|\left(1-B k^{2}\right)$ is the actual Hele-Shaw growth rate-Eq. (4.13) fixes $a \pm$ to be

$$
a_{ \pm}=\frac{i \omega}{k}\left[1-\epsilon|k| \frac{\sqrt{2}}{2} g_{ \pm}(c) \frac{p_{+}+p_{-}}{2}\right] .
$$

Finally, Eq. (4.12) requires that the following dispersion relation is satisfied:

$$
\begin{aligned}
\omega= & \frac{\omega_{0}}{\left[(1+c) p_{-}+(1-c) p_{+}\right] / 2}\left[1+\epsilon|k| \frac{\sqrt{2}}{2} \frac{p_{-}+p_{+}}{2}\right. \\
& \left.\times \frac{g_{+}(1-c) p_{+}+g_{-}(1+c) p_{-}}{(1-c) p_{+}+(1+c) p_{-}}\right]+O\left(\epsilon^{2}\right) \\
= & \omega_{0}\left(\frac{1}{\sqrt{1+\left(\widetilde{\epsilon} \omega / k^{2}\right)}}-\epsilon|k| \sqrt{2} \frac{5}{6}\right)+O\left(c^{2}\right)+O\left(\epsilon^{2}\right) .
\end{aligned}
$$

This consists of the well known Hele-Shaw growth rate multiplied by a factor smaller than 1 , carrying the corrections in $\epsilon$ and $\widetilde{\epsilon}$. We identify the conditions on $\epsilon$ and $\widetilde{\epsilon}$ heuristically derived at the beginning of Sec. IV to control how close this factor is to 1, and in general how close the stream function is to the actual Hele-Shaw one: $\widetilde{\epsilon} \omega / k^{2} \ll 1 \pm c$ (within $p_{ \pm}$) and $\epsilon|k| \ll 1$ in Eqs. (5.2)-(5.4), and the simplified version up to $O(c) \tilde{\epsilon} \omega / k^{2} \ll 1$ and $\epsilon|k| \ll 1$ in Eq. (5.5). The amplitude factor [Eq. (5.3)] can also be expanded in powers of $c$ making use of Eq. (5.5) to find

$$
a_{ \pm}=\frac{i \omega_{0}}{k}\left(\frac{1}{\sqrt{1+\left(\widetilde{\boldsymbol{\epsilon}} w / k^{2}\right)}} \mp c \epsilon|k| \sqrt{2}\right)+O\left(c^{2}\right)+O\left(\epsilon^{2}\right) .
$$

Since these corrections have a stabilizing effect, they could affect the selection of the steady finger width. As a matter of fact, Ben Amar already showed that the $\partial_{s}(\hat{y} \cdot \hat{s})$ term of $\Gamma_{s}$ in Eq. (4.13) on its own was capable of selecting a finger width greater than $\frac{1}{2}$ [22]. Then, for small enough values of the physical surface tension (i.e., the physical selection mechanism), for which a width very close to $\frac{1}{2}$ should be expected, this term could turn out to control the selection itself, so that an unexpected greater width could be obtained. Of course, this will not be the case if a sufficiently small value of the interface thickness $\epsilon$ is used, so that the condition $\epsilon|k| \ll 1$ is satisfied for the length scale set by the surface tension: $\epsilon \ll \sqrt{B}$.

\section{CONCLUSIONS}

We have introduced a phase-field model for Hele-Shaw flows with arbitrary viscosity contrast, and shown it to yield the proper sharp-interface limit. We have actually found two independent small parameters $(\epsilon$ and $\widetilde{\epsilon})$ and three distinct conditions on them to control the convergence to the sharpinterface limit $\epsilon, \widetilde{\epsilon} \rightarrow 0$. In particular, $\widetilde{\epsilon}$ must be lowered when $c$ is increased. A thin-interface model, i.e., an effective sharp interface model keeping finite- $\boldsymbol{\epsilon}$ and $-\tilde{\boldsymbol{\epsilon}}$ effects, has been derived for the dynamics of the phase-field model up to first order in both of these parameters. This thin-interface model has then been used to compute the finite- $\epsilon$ and $-\widetilde{\epsilon}$ corrections to the Hele-Shaw result explicitly for a specific situation such as the linear regime, thus suggesting that the singlefinger width selection could also be affected by these finitethickness effects.

In the following paper [23] we perform numerical simulations of the phase-field model [Eqs. (2.7) and (2.8)], and we explicitly vary the two small parameters $\epsilon$ and $\widetilde{\boldsymbol{\epsilon}}$ independently. In this way we both control the simulation accuracy through the conditions mentioned to show how to reproduce the Hele-Shaw dynamics within this method, and explicitly check convergence in the interface thickness.

\section{ACKNOWLEDGMENTS}

We are indebted to J. L. Mozos, E. Corvera, and H. Guo for collaboration in the early stages of this work. We acknowledge financial support from the Dirección General de Enseñanza Superior (Spain), under Projects No. PB96-1001C02-02, PB96-0378-C02-01, and PB96-0241-C02-02, and the European Commission Project No. ERB FMRX-CT960085. R.F. also acknowledges a grant from the Comissionat per a Universitats i Recerca (Generalitat de Catalunya).

\section{APPENDIX}

Our goal here is to rescale the differential operators appearing in the phase-field model [Eqs. (2.7) and (2.8)]. The first step will be to rewrite them in terms of the local coordinates defined on the interface $r$ and $s$. To do this, one must precisely define the curvilinear coordinate system and compute its so-called scale factors:

Consider the $\theta=0$ level set and its intrinsic coordinates $s$ (the arclength along it) and $r$ (the signed distance to it, positive for a point with $\theta>0$ ), so that $\hat{s} \times \hat{r}=\hat{x} \times \hat{y}$. Let $\alpha$ be the angle going from $\hat{x}$ to $\hat{s}$. Then $\kappa=\alpha_{s}$ is the $\theta=0$ level-set curvature. We introduce $X$ and $Y$ as the values of $x$ and $y$ for a point on the $\theta=0$ curve with a given value of $s$. By moving this point infinitesimally along $s$ we find that these values have changed in $d Y=d s \sin \alpha, d X=d s \cos \alpha$. Consider also the coordinates $x$ and $y$ of a point with $\theta \neq 0$ in terms of the values $X$ and $Y$ of its closest neighbor on the $\theta=0$ level set, and the signed distance between them. Taking into account that $\alpha$ is also the angle going from $\hat{y}$ to $\hat{r}$, one finds $x=X$ $-r \sin \alpha$ and $y=Y+r \cos \alpha$. Now one can compute the 
(positive defined) scale factors

$$
\begin{gathered}
h_{r}^{2} \equiv x_{r}^{2}+y_{r}^{2}=1 \Rightarrow h_{r}=1, \\
h_{s}^{2} \equiv x_{s}^{2}+y_{s}^{2}=\left(X_{s}-r \alpha_{s} \cos \alpha\right)^{2}+\left(Y_{s}-r \alpha_{s} \sin \alpha\right)^{2} \\
=(\cos \alpha-r \kappa \cos \alpha)^{2}+(\sin \alpha-r \kappa \sin \alpha)^{2} \\
=(1-r \kappa)^{2} \Rightarrow h_{s}=|1-r \kappa|=1-r \kappa .
\end{gathered}
$$

Note that the last equality in Eq. (A2) requires that $r \kappa<1$. In the inner region, where we make use of such formulas, this will hold as long as the interface thickness $\epsilon$ is much smaller than the curvature radius at any point of the interface, i.e., not too far from the sharp-interface limit. Otherwise the present analysis would break down, because one could always find a point such that $r \kappa=1$, where $h_{s}$ would vanish, reflecting the fact that the change of coordinates has become ambiguous in $s$.

Then, the scale factors are used to express the differential operators in terms of $r$ and $s$ :

$$
\begin{gathered}
\vec{\nabla} a=a_{r} \hat{r}+\frac{a_{s}}{1-r \kappa} \hat{s}, \\
\vec{\nabla} \cdot \vec{a}=\left(a^{r}\right)_{r}+\frac{-\kappa a^{r}+\left(a^{s}\right)_{s}}{1-r \kappa} \quad\left(\vec{a}=a^{r} \hat{r}+a^{s} \hat{s}\right), \\
\nabla^{2} a=a_{r r}-\frac{\kappa a_{r}}{1-r \kappa}+\frac{a_{s s}}{(1-r \kappa)^{2}}+\frac{r \kappa_{s} a_{s}}{(1-r \kappa)^{3}} .
\end{gathered}
$$

Finally, one sets $r=\epsilon \rho$ and expands in powers of $\epsilon$ :

$$
(1-r \kappa)^{-1}=1+\epsilon \rho \kappa+O\left(\epsilon^{2}\right) .
$$

One obtains

$$
\begin{gathered}
\vec{\nabla} a=\frac{1}{\epsilon} A_{\rho} \hat{r}+\hat{s} A_{s}\left[1+\epsilon \rho \kappa+O\left(\epsilon^{2}\right)\right], \\
\vec{\nabla} \cdot \vec{a}=\frac{1}{\epsilon}\left(A^{r}\right) \rho+\left[\left(A^{s}\right)_{s}-\kappa A^{r}\right]\left[1+\epsilon \rho \kappa+O\left(\epsilon^{2}\right)\right], \\
\nabla^{2} a=\frac{1}{\epsilon^{2}} A_{\rho \rho}-\frac{1}{\epsilon} \kappa A_{\rho}-\rho \kappa^{2} A_{\rho}+A_{s s}+O(\epsilon) .
\end{gathered}
$$

This completes the rescaling. Capital letters denote fields written in the rescaled coordinates of the inner region. Any other quantity appearing in Eqs. (2.7) and (2.8) is derived from these. For instance, we obtain

$$
\begin{aligned}
\frac{\partial a}{\partial t} & \left.\equiv \frac{\partial a}{\partial t}\right|_{x, y=\text { const }}=\frac{d a}{d t}-\overrightarrow{\mathrm{v}} \cdot \vec{\nabla} a \\
& =-\frac{\mathrm{v}_{n}}{\epsilon} A_{\rho}+\frac{d a}{d t}-\mathrm{v}_{t} A_{s}+O(\epsilon),
\end{aligned}
$$

where the partial (total) time derivative is computed keeping $x$ and $y(r$ and $s$ ) fixed, and $\vec{v}$ is the velocity of the $r, s$ frame with respect to the $x, y$ one, i.e., the interface velocity. Moreover,

$$
\begin{aligned}
\hat{z} \cdot(\vec{\nabla} \psi \times \vec{\nabla} \theta)= & \frac{1}{\epsilon}\left\{\Psi_{s} \Theta_{\rho}\left[1+\epsilon \rho \kappa+O\left(\epsilon^{2}\right)\right]\right. \\
& \left.-\Psi_{\rho} \Theta_{s}[1+O(\epsilon)]\right\} \\
\vec{\nabla} \cdot(\theta \vec{\nabla} \psi)= & \frac{1}{\epsilon^{2}}\left(\Theta \Psi_{\rho}\right)_{\rho}+\left(\Theta \Psi_{s}\right)_{s}-\frac{1}{\epsilon} \kappa \Theta \Psi_{\rho}(1+\epsilon \rho \kappa) \\
& +O(\epsilon) \\
= & \frac{1}{\epsilon^{2}}\left(\Theta \Psi_{\rho}\right)_{\rho}-\frac{1}{\epsilon} \kappa \Theta \Psi_{\rho}-\rho \kappa^{2} \Theta \Psi_{\rho}+\left(\Theta \Psi_{s}\right)_{s} \\
& +O(\epsilon) .
\end{aligned}
$$

The only terms left in Eqs. (2.7) and (2.8) to compute are those containing $\gamma(\theta)$ and $\kappa(\theta)$. To construct them we will need the following quantities:

$$
\begin{aligned}
|\vec{\nabla} \theta| & =+\sqrt{\frac{1}{\epsilon^{2}} \Theta_{\rho}^{2}+\Theta_{s}^{2}[1+O(\epsilon)]^{2}} \\
& =+\frac{\Theta_{\rho}}{\epsilon} \sqrt{1+\epsilon^{2} \frac{\Theta_{s}^{2}}{\Theta_{\rho}^{2}}[1+O(\epsilon)]^{2}} \\
& =+\frac{\Theta_{\rho}}{\epsilon}\left[1+\frac{\epsilon^{2}}{2} \frac{\Theta_{s}^{2}}{\Theta_{\rho}^{2}}+O\left(\epsilon^{3}\right)\right] \\
& =\frac{\Theta_{\rho}}{\epsilon}+\frac{\epsilon}{2} \frac{\Theta_{s}^{2}}{\Theta_{\rho}}+O\left(\epsilon^{2}\right) .
\end{aligned}
$$

(note that $\Theta_{\rho}>0$, since $\Theta$ is monotonic in $\rho$ and we defined $r$ to be positive for the $\theta>0$ phase) and

$$
\begin{aligned}
\hat{r}(\theta) & \equiv \frac{\vec{\nabla} \theta}{|\vec{\nabla} \theta|}=\frac{\epsilon / \Theta_{\rho}}{\epsilon / \Theta_{\rho}} \times \frac{\vec{\nabla} \theta}{|\vec{\nabla} \theta|}=\frac{\hat{r}+\hat{s} \epsilon\left(\Theta_{s} / \Theta_{\rho}\right)[1+O(\epsilon)]}{1+\epsilon^{2} \frac{1}{2}\left(\Theta_{s}^{2} / \Theta_{\rho}^{2}\right)+O\left(\epsilon^{3}\right)} \\
& =\hat{r}\left[1-\frac{\epsilon^{2}}{2} \frac{\Theta_{s}^{2}}{\Theta_{\rho}^{2}}+O\left(\epsilon^{3}\right)\right]+\hat{s}\left[\epsilon \frac{\Theta_{s}}{\Theta_{\rho}}+O\left(\epsilon^{2}\right)\right] .
\end{aligned}
$$

We have termed this $\hat{r}(\theta)$ because it is indeed the unit vector normal to the $\theta=$ const level set on which it is computed. We similarly define $\hat{s}(\theta) \equiv \hat{r}(\theta) \times \hat{z}$ and $\kappa(\theta) \equiv-\vec{\nabla} \cdot(\vec{\nabla} \theta /|\vec{\nabla} \theta|)$ to construct $\gamma(\theta) / 2 \equiv B \hat{s}(\theta) \cdot \vec{\nabla}_{\kappa}(\theta)+\hat{y} \cdot \hat{s}(\theta)$ : 


$$
\begin{aligned}
-\kappa(\theta) & \equiv \vec{\nabla} \cdot \frac{\vec{\nabla} \theta}{|\vec{\nabla} \theta|} \\
& =-\frac{\epsilon}{2}\left(\frac{\Theta_{s}^{2}}{\Theta_{\rho}^{2}}\right)_{\rho}+\epsilon\left(\frac{\Theta_{s}}{\Theta_{\rho}}\right)_{s}-\kappa(1+\epsilon \rho \kappa)+O\left(\epsilon^{2}\right) \\
& =-\kappa+\epsilon\left[\left(\frac{\Theta_{s}}{\Theta_{\rho}}\right)_{s}-\frac{1}{2}\left(\frac{\Theta_{s}^{2}}{\Theta_{\rho}^{2}}\right)_{\rho}-\rho \kappa^{2}\right]+O\left(\epsilon^{2}\right),
\end{aligned}
$$

$$
\begin{aligned}
\vec{\nabla} \kappa(\theta)= & -\hat{r}\left[\left(\frac{\Theta_{s}}{\Theta_{\rho}}\right)_{s \rho}-\frac{1}{2}\left(\frac{\Theta_{s}^{2}}{\Theta_{\rho}^{2}}\right)_{\rho \rho}-\kappa^{2}+O(\epsilon)\right] \\
& +\hat{s}\left\{\kappa_{s}+\epsilon\left[-\left(\frac{\Theta_{s}}{\Theta_{\rho}}\right)_{s s}+\frac{1}{2}\left(\frac{\Theta_{s}^{2}}{\Theta_{\rho}^{2}}\right)_{\rho s}+2 \rho \kappa \kappa_{s}\right]\right. \\
& \left.+O\left(\epsilon^{2}\right)\right\}\left[1+\epsilon \rho \kappa+O\left(\epsilon^{2}\right)\right] \\
= & -\hat{r}\left[-\kappa^{2}+\left(\frac{\Theta_{s}}{\Theta_{\rho}}\right)_{s \rho}-\frac{1}{2}\left(\frac{\Theta_{s}^{2}}{\Theta_{\rho}^{2}}\right)+O(\epsilon)\right] \\
& +\hat{s}\left\{\kappa_{s}+\epsilon\left[3 \rho \kappa \kappa_{s}-\left(\frac{\Theta_{s}}{\Theta_{\rho}}\right)_{s s}+\frac{1}{2}\left(\frac{\Theta_{s}^{2}}{\Theta_{\rho}^{2}}\right)_{\rho s}\right]\right. \\
& \left.+O\left(\epsilon^{2}\right)\right\},
\end{aligned}
$$

$$
\begin{aligned}
\frac{\gamma(\theta)}{2}= & B\left\{\kappa_{s}+\epsilon\left[3 \rho \kappa \kappa_{s}-\left(\frac{\Theta_{s}}{\Theta_{\rho}}\right)_{s s}+\frac{1}{2}\left(\frac{\Theta_{s}^{2}}{\Theta_{\rho}^{2}}\right)_{\rho s}\right]\right. \\
& \left.+\epsilon \frac{\Theta_{s}}{\Theta_{\rho}}\left[-\kappa^{2}+\left(\frac{\Theta_{s}}{\Theta_{\rho}}\right)_{s \rho}-\frac{1}{2}\left(\frac{\Theta_{s}^{2}}{\Theta_{\rho}^{2}}\right)_{\rho \rho}\right]\right\} \\
& +\hat{y} \cdot \hat{s}-\epsilon \frac{\Theta_{s}}{\Theta_{\rho}} \hat{y} \cdot \hat{r}+O\left(\epsilon^{2}\right) \\
= & \frac{\gamma}{2}+O(\epsilon) .
\end{aligned}
$$

We should still compute the product $\kappa(\theta)|\vec{\nabla} \theta|$ appearing in Eq. (2.8), but instead we prefer to compute straight ahead the $\operatorname{sum} \quad \nabla^{2} \theta+\kappa(\theta)|\vec{\nabla} \theta|=\nabla^{2} \theta-\nabla^{2} \theta+(\vec{\nabla} \theta /|\vec{\nabla} \theta|) \cdot \vec{\nabla}|\vec{\nabla} \theta|$ $=\hat{r}(\theta) \cdot \vec{\nabla}|\vec{\nabla} \theta|:$

$$
\begin{aligned}
& \vec{\nabla}|\vec{\nabla} \theta|=\hat{r}\left[\frac{\Theta_{\rho \rho}}{\epsilon^{2}}+\frac{1}{2}\left(\frac{\Theta_{s}^{2}}{\Theta_{\rho}}\right)_{\rho}+O(\epsilon)\right]+\hat{s}\left[\frac{\Theta_{\rho s}}{\epsilon}+O\left(\epsilon^{0}\right)\right], \\
& \nabla^{2} \theta+\kappa(\theta)|\vec{\nabla} \theta|= \frac{\Theta_{\rho \rho}}{\epsilon^{2}}+\frac{1}{2}\left[\left(\frac{\Theta_{s}^{2}}{\Theta_{\rho}}\right)-\frac{\Theta_{s}^{2}}{\Theta_{\rho}^{2}} \Theta_{\rho \rho}+2 \frac{\Theta_{s}}{\Theta_{\rho}} \Theta_{\rho s}\right] \\
&+O(\epsilon) \\
&= \frac{\Theta_{\rho \rho}}{\epsilon^{2}}+\frac{\Theta_{s} \Theta_{\rho s}}{\Theta_{\rho}}\left(1+\frac{1}{\Theta_{\rho}}\right)-\frac{\Theta_{s}^{2} \Theta_{\rho \rho}}{\Theta_{\rho}^{2}} \\
&+O(\epsilon) .
\end{aligned}
$$

[1] J. S. Langer, Rev. Mod. Phys. 52, 1 (1980); D. A. Kessler, J. Koplik, and H. Levine, Adv. Phys. 35, 255 (1988); P. Pelcé, Perspectives in Physics (Academic, New York, 1988).

[2] P. G. Saffman and G. I. Taylor, Proc. R. Soc. London, Ser. A 245, 312 (1958); D. Bensimon, L. Kadanoff, S. Liang, B. I. Shraiman, and C. Tang, Rev. Mod. Phys. 58, 977 (1986).

[3] M. Siegel and S. Tanveer, Phys. Rev. Lett. 76, 419 (1996); F. X. Magdaleno and J. Casademunt, Phys. Rev. E 57, R3707 (1998).

[4] G. Tryggvason and H. Aref, J. Fluid Mech. 136, 1 (1983).

[5] G. Tryggvason and H. Aref, J. Fluid Mech. 154, 287 (1985).

[6] J. Casademunt, A. Hernández-Machado, and D. Jasnow, Int. J. Mod. Phys. B 6, 1647 (1992).

[7] J. Casademunt and D. Jasnow, Phys. Rev. Lett. 67, 3677 (1991); Phys. Rev. D 79, 387 (1994).

[8] D. Jasnow and J. Viñals, Phys. Rev. A 40, 3864 (1989); 41, 6910 (1990).

[9] T. Y. Hou, J. S. Lowengrub, and M. J. Shelley, J. Comput. Phys. 114, 312 (1994); M. J. Shelley, J. Fluid Mech. 244, 493 (1992); L. Kondic, M. J. Shelley, and P. Palffy-Muhoray, Phys. Rev. Lett. 80, 1433 (1998).

[10] J. B. Collins and H. Levine, Phys. Rev. B 31, 6119 (1985); J. S. Langer, in Directions in Condensed Matter Physics, edited by G. Grinstein and G. Mazenko (World Scientific, Singapore, 1986), p. 165.
[11] R. Kobayashi, Physica D 63, 410 (1993).

[12] G. McFadden, A. Wheeler, R. Braun, S. Coriell, and R. Sekerka, Phys. Rev. E 48, 2016 (1993); A. Wheeler, B. Murray, and R. Schaefer, Physica D 66, 243 (1993); B. Murray, A. Wheeler, and M. Glicksman, J. Cryst. Growth 154, 386 (1995); S. Wang and R. Sekerka, Phys. Rev. E 53, 3760 (1996).

[13] A. Karma and W-J. Rappel, Phys. Rev. E 53, 3017 (1996); Phys. Rev. Lett. 77, 4050 (1996); Phys. Rev. E 57, 4323 (1998).

[14] S. Wang, R. Sekerka, A. Wheeler, B. Murray, S. Coriell, R. Braun, and G. McFadden, Physica D 69, 189 (1993).

[15] K. Elder, F. Drolet, J. Kosterlitz, and M. Grant, Phys. Rev. Lett. 72, 677 (1994).

[16] A. Bosch, H. Müller-Krumbhaar, and O. Shochet, Z. Phys. B 97, 367 (1995); T. Abel, E. Brener, and H. Müller-Krumbhaar, Phys. Rev. E 55, 7789 (1997).

[17] J.-L. Mozos and H. Guo, Europhys. Lett. 32, 61 (1995).

[18] R. González-Cinca, L. Ramírez-Piscina, J. Casademunt, A. Hernández-Machado, L. Kramer, T. T. Katona, T. Börzsönyi, and A. Buka, Physica D 99, 359 (1996); T. T. Katona, T. Börzsönyi, Z. Váradi, J. Szabon, A. Buka, R. González-Cinca, L. Ramírez-Piscina, J. Casademunt, and Hernández-Machado, Phys. Rev. E 54, 1574 (1996); R. González-Cinca, L. Ramírez- 
Piscina, J. Casademunt, A. Hernández-Machado, T. T. Katona, T. Börzsönyi, and A. Buka, J. Cryst. Growth 193, 712 (1998). [19] G. Caginalp, Phys. Rev. A 39, 5887 (1989).

[20] P. C. Hohenberg and B. I. Halperin, Rev. Mod. Phys. 49, 435 (1977).
[21] J. V. Maher, Phys. Rev. Lett. 54, 1498 (1985).

[22] M. Ben Amar, Phys. Fluids 4, 2641 (1992).

[23] R. Folch, J. Casademunt, A. Hernández-Machado, and L. Ramírez-Piscina, following paper, Phys. Rev. E 60, 1734 (1999). 